\title{
Dampening Controllers via a Riccati Equation Approach*
}

\author{
J.J. Hench ${ }^{\dagger} \quad$ C. $\mathrm{He}^{\ddagger} \quad$ V. Kučera ${ }^{\dagger} \quad$ V. Mehrmann ${ }^{\ddagger}$ \\ ${ }^{\dagger}$ Institute of Information Theory and Automation \\ Academy of Sciences of the Czech Republic \\ Pod vodárenskou věží 4 \\ 12808 Praha 8 \\ Czech Republic \\ $\ddagger$ Technische Universität Chemnitz-Zwickau \\ Fakultät für Mathematik \\ D-09107 Chemnitz \\ Germany \\ $e$-mail: \\ hench@utia.cas.cz \\ kucera@utia.cas.cz \\ heomathematik.tu-chemnitz.de \\ mehrmann@mathematik.tu-chemnitz.de
}

May 22, 1995

*Support for this research was provided by the Academy of Sciences of the Czech Republic, by the Grant Agency of the Czech Republic under contract number 102/94/0294, and by the Deutsche Forschungsgemeinschaft Projekt Singuläre Steuerungsprobleme, contract number ME-790/7-1 


\begin{abstract}
An algorithm is presented which computes a state feedback for a standard linear system which not only stabilizes, but also dampens the closed-loop system dynamics. In other words, a feedback gain vector is computed such that the eigenvalues of the closed-loop state matrix are within the region of the left half-plane where the magnitude of the real part of each eigenvalue is greater than the imaginary part. This may be accomplished by solving one periodic algebraic Riccati equation and one degenerate Riccati equation. The solution to these equations are computed using numerically robust algorithms.

Finally, the periodic Riccati equation is unusual in that it produces one symmetric and one skew symmetric solution, and as a result two different state feedbacks. Both feedbacks dampen the system dynamics, but produce different closed-loop eigenvalues, giving the controller designer greater freedom in choosing a desired feedback.
\end{abstract}

\title{
Keywords
}

linear quadratic controller, dampening feedback, damped dynamics, periodic systems, periodic Riccati equation.

\section{Introduction}

Obtaining a stabilizing controller for a standard linear time-invariant system is a rather straight-forward problem; all that is necessary to produce a stabilizing controller is the stabilizing solution of an algebraic Riccati equation, or in a simplified case, a Lyapunov equation. In practice, however, such a stabilizing feedback is often undesirable for the simple reason that stability may be too weak a constraint. Often what is desired is a controller which guarantees that the closed-loop dynamics are damped. Simple solutions to this requirement have not previously existed. Increased relative weighting of the input versus the state (or output) in the quadratic cost function of the Linear Quadratic Regulator problem often has little effect on the damping factor of the optimal feedback; it tends to move the poles of the closed-loop system further away from both the real and the imaginary axis. Another method, devised by Anderson and Moore [1] introduces a shift into the algebraic Riccati equation. The effect of this is to move the poles of the closed-loop system away from the imaginary axis, but does not necessarily guarantee that the closed-loop dynamics are damped. The end result is that often the control engineer is forced to place the poles of the 
closed-loop system to achieve the required damping. Unfortunately, poleplacement is usually an inherently ill-conditioned problem [7], and becomes impractical for large-order systems [8].

To circumvent these difficulties, we derive a new method which stabilizes a linear system such that the dynamics of the closed-loop system are damped, i.e., that the real part of each of the eigenvalues of the closedloop system matrix is greater in magnitude than the imaginary part. This is accomplished, in part, by computing the solution to a particular periodic algebraic Riccati equation which moves the poles from outside to inside the union of the damped region and anti-damped regions of the complex plane. This is based on the observation that eigenvalues of a matrix in these damped regions are anti-stable when the matrix is squared. If a periodic system is used to describe the negative square of the closed-loop system, then the proposed periodic Riccati equation can be used to stabilize this system.

This method has a number of interesting properties. First, the algorithm produces two different Riccati solutions: one symmetric and one skew symmetric. While both of these solutions produce a dampening feedback, they have different properties. Second, the method described in this paper may be used in conjunction with the standard stabilization methods via the solution of Lyapunov or Riccati equations, such as those mentioned in [8], and Anderson and Moore's shifting method [1] to restrict the poles of the closed-loop system to a more complex region in the left half-plane. Third, all of the feedbacks mentioned may be computed using Schur methods to compute invariant subspaces $[10,14]$. For these methods numerical robustness has been demonstrated. Fourth, by varying the periodicity of the aforementioned periodic Riccati equation, it is possible to restrict the poles of the closed-loop system to ever narrower cones in the left half-plane. Finally, the dampening controller may be viewed as a controller that results from a particular choice of the state weighting matrix in the quadratic cost function of the standard linear quadratic regulator problem. This state weighting matrix provides valuable information about the states that need to be weighted more heavily in order to produce a dampening controller. In addition, the close connection of the method of this paper to the usual algebraic Riccati equation avails it to standard techniques of the analysis of matrix quadratic equations $[12,13]$. 


\section{Damped Riccati Equations}

Throughout this paper, we will be concerned with the computation of a feedback $u(t)$ which stabilizes the standard linear time-invariant system

$$
\dot{x}=A x+B u
$$

Here $A \in \mathbb{R}^{n, n}, B \in \mathbb{R}^{n, m}$, and we assume that the matrix pair $\langle A, B\rangle$ is reachable, (i.e. $\operatorname{Rank}[\lambda I-A, B]=n$ for all complex $\lambda$ ). Furthermore, we require the feedback to be proportional to the state $x$, i.e.,

$$
u(t)=F x(t) .
$$

A wide range of stabilizing feedbacks of this form may be obtained by the solution of an algebraic Riccati equation $[11,13]$. Given any matrix $C \in \mathbb{R}^{p, n}$ such that the matrix pair $\langle A, C\rangle$ is observable, (i.e., $\left\langle A^{T}, C^{T}\right\rangle$ is reachable), then a stabilizing feedback in (2) is obtained as

$$
u(t)=-B^{T} W x(t),
$$

where $W$ is the symmetric positive semi-definite solution of the algebraic Riccati equation ( $A R E$ )

$$
0=A^{T} W+W A-W B B^{T} W+C^{T} C,
$$

see $[4,8,14,20]$. An integral part of methods which compute an (optimal) stabilizing controller is the computation of a basis for an appropriate invariant subspace of a related Hamiltonian system $[4,14,18,21]$. If the columns of the $2 n \times n$ matrix

$$
T=\left[\begin{array}{l}
T_{11} \\
T_{21}
\end{array}\right]
$$

span the invariant subspace corresponding to the stable eigenvalues of the $2 n \times 2 n$ Hamiltonian matrix

$$
H=\left[\begin{array}{cc}
A & -B B^{T} \\
-C^{T} C & -A^{T}
\end{array}\right],
$$

and if $T_{11}$ is invertible, then the symmetric positive semi-definite solution $W$ of the algebraic Riccati equation in (4) is given by $W=T_{21} T_{11}^{-1},[14,20]$.

The computation of a dampening feedback can be carried out along the same lines. First, we introduce a linear zero-sum non-cooperative dynamic 
game from which arise two Riccati equations, whose solutions provide the required dampening feedback. As mentioned in the introduction, dampening feedback requires, in a manner of speaking, stabilization of the squares of the state matrix. By defining the square of the state matrix as the state matrix of this linear dynamic game, and by appropriately choosing the input and output matrices and the quadratic cost function, the resulting minimax problem can be shown to be equivalent to a stable invariant subspace identification problem for a periodic Hamiltonian system. The periodicity is essential in that it indirectly produces a stability region that is not the left-half plane, but rather a pair of cones in the complex plane, as shown by Figure 1.

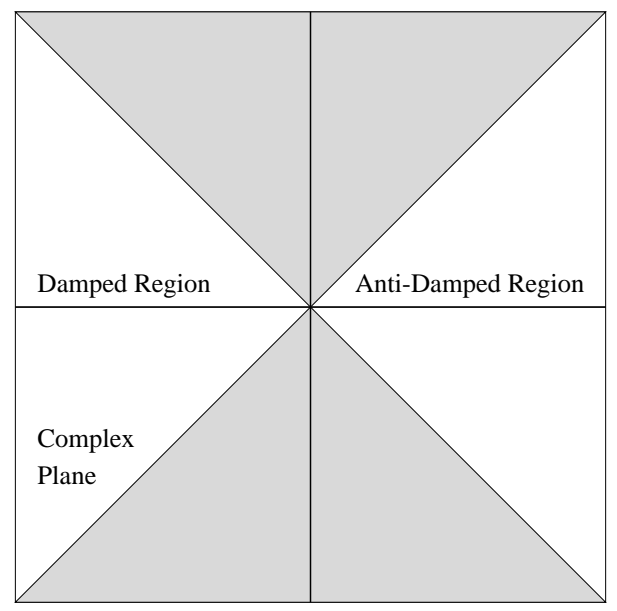

Figure 1: Damped Stability Region

Proceeding, we examine the following linear dynamic game. Consider linear systems of the form

$$
\begin{aligned}
\dot{z} & =-\left(A-B B^{T} C^{T} C\right)^{2} z+(A-I) B u+(A+I) B v \quad, \quad z(0)=z_{0} \\
y & =C(A-I) z \\
w & =C(A+I) z
\end{aligned}
$$

with quadratic cost functional

$$
\min _{u} \max _{v} \frac{1}{2} \int_{0}^{\infty}\left[y^{T} y-w^{T} w+u^{T} u-v^{T} v\right] d t
$$

where the matrices $A \in \mathbb{R}^{n, n}, B \in \mathbb{R}^{n, m}, C \in \mathbb{R}^{p, n}$. The open-loop Nash equilibrium may be computed via the Hamilton-Jacobi-Bellman (or Issac's) 
equation [2], which gives rise to a two-point boundary problem and a linear differential equation in the state $z$ and costate $q$

$$
\left[\begin{array}{c}
\dot{z} \\
\dot{q}
\end{array}\right]=\left[\begin{array}{cc}
-(A-R S)^{2} & A R+R A^{T} \\
S A+A^{T} S & (A-R S)^{2 T}
\end{array}\right]\left[\begin{array}{l}
z \\
q
\end{array}\right]
$$

with

$$
\begin{aligned}
z(0) & =z_{0} \\
q(\infty) & =0, \\
u & =-B^{T}\left(A^{T}-I\right) q, \\
v & =B^{T}\left(A^{T}+I\right) q, \\
R & =B B^{T}, \\
S & =C^{T} C .
\end{aligned}
$$

The linear differential equation in (9) may be factored into a product form:

$$
\begin{aligned}
& {\left[\begin{array}{c}
\dot{z} \\
\dot{q}
\end{array}\right]=\left[\begin{array}{cc}
-A & R \\
S & A^{T}
\end{array}\right] \quad\left[\begin{array}{cc}
A & -R \\
S & A^{T}
\end{array}\right] \quad\left[\begin{array}{l}
z \\
q
\end{array}\right]} \\
& =\begin{array}{lll}
H_{2} & H_{1}
\end{array}\left[\begin{array}{l}
z \\
q
\end{array}\right] \\
& =\quad H_{X} \quad\left[\begin{array}{l}
z \\
q
\end{array}\right],
\end{aligned}
$$

where

$$
H_{X}=\left[\begin{array}{cc}
-\left(A^{2}-R S\right) & A R+R A^{T} \\
S A+A^{T} S & \left(A^{2}-R S\right)^{T}
\end{array}\right]
$$

Since the matrix $H_{X}$ is Hamiltonian, a Riccati equation is associated with it. In addition, since $H_{X}$ may be written as the product $H_{2} H_{1}$, it is possible to write a related Hamiltonian system with

$$
H_{Y}=\left[\begin{array}{cc}
-\left(A^{2}+R S\right) & A R-R A^{T} \\
S A-A^{T} S & \left(A^{2}+R S\right)^{T}
\end{array}\right],
$$

which is the product $H_{1} H_{2}$. As with $H_{X}$, this latter system will give rise to a different but related Riccati equation. These two Riccati equations are denoted as the Symmetric Damped Algebraic Riccati Equation (SDARE) and the Skew-Symmetric Damped Algebraic Riccati Equation (SSDARE), respectively, and are formally described in the following definition. 


\section{Definition 1}

Let $A, R, S$ and $I \in \mathbb{R}^{n, n}$ with $R=B B^{T}, S=C^{T} C, I$ be the identity matrix, and $\sigma \in \mathbb{R}$. Then

a) the Algebraic Riccati Equation (ARE) is defined as

$$
W A+A^{T} W-W R W+S=0,
$$

b) the Shifted Algebraic Riccati Equation (SHARE) is defined as

$$
Z(A+\sigma I)+\left(A^{T}+\sigma I\right) Z-Z R Z+S=0,
$$

c) the Symmetric Damped Algebraic Riccati Equation (SDARE) is defined as

$$
X\left(A^{2}-R S\right)+\left(A^{2 T}-S R\right) X-X\left(A R+R A^{T}\right) X+\left(A^{T} S+S A\right)=0 .
$$

and

d) the Skew-Symmetric Damped Algebraic Riccati Equation (SSDARE) is defined as

$$
Y\left(A^{2}+R S\right)+\left(A^{2 T}+S R\right) Y-Y\left(A R-R A^{T}\right) Y+\left(A^{T} S-S A\right)=0 .
$$

The solutions to the Symmetric Damped Algebraic Riccati Equation and the Skew-Symmetric Damped Algebraic Riccati Equation are intimately connected with the Riccati solution of the standard Algebraic Riccati Equation and optimal stabilizing feedback, as we demonstrate in the following lemmas and theorems.

\section{Theorem 1}

a) Suppose that the matrix pair $\left\langle-A^{2}+R S, A R+R A^{T}\right\rangle$ is reachable and that $A R+R A^{T}$ is negative semi-definite. Then the following are equivalent:

i) There exists a Hermitian matrix $X$ such that (16) holds.

ii) There exists an Hermitian solution $X_{1}$ of (16) such that the closed-loop matrix $-A^{2}+R S+\left(A R+R A^{T}\right) X_{1}$ is semi-stable, i.e., all eigenvalues are in the closed left half-plane. 
iii) The partial multiplicities of all purely imaginary eigenvalues (if any) of the Hamiltonian matrix $H_{X}$ are all even.

b) Suppose that the matrix pair $\left\langle-A^{2}-R S, A R-R A^{T}\right\rangle$ is reachable and that $\imath\left(A R-R A^{T}\right)(\imath=\sqrt{-1})$ is Hermitian negative semi-definite. Then the following are equivalent:

i) There exists a skew-Hermitian matrix $Y$ such that (17) holds.

ii) There exists a skew-Hermitian solution $Y_{1}$ of (17) such that the closed-loop matrix $-A^{2}-R S+\left(A R-R A^{T}\right) Y_{1}$ is semi-stable, i.e., all eigenvalues are in the closed left half-plane.

iii) The partial multiplicities of all purely imaginary eigenvalues (if any) of the matrix $H_{Y}$ are all even.

Proof. Part a) is Theorem 1 in [15]. Part b) follows directly from a), since $H_{Y}$ is similar to the complex Hamiltonian matrix

$$
\tilde{H}_{Y}=\left[\begin{array}{cc}
-\left(A^{2}+R S\right) & \imath\left(A R-R A^{T}\right) \\
\imath\left(S A-A^{T} S\right) & \left(A^{2}+R S\right)^{T}
\end{array}\right] .
$$

Note that this existence result yields only the existence of Hermitian solutions, which may be complex. The existence of real symmetric solutions is closely related to the existence of Hermitian solutions, see [16]. We refrain here from discussing this relationship in detail. For our purpose it is important to discuss the symmetric stabilizing solution, which is indeed real.

\section{Corollary 2}

a) Suppose that the matrix pair $\left\langle-A^{2}+R S, A R+R A^{T}\right\rangle$ is reachable and that $A R+R A^{T}$ is negative semi-definite. Then there exists a real symmetric matrix solution $X$ of (16) such that the closed-loop matrix $-A^{2}+R S+\left(A R+R A^{T}\right) X$ is stable, i.e., all eigenvalues are in the open left half plane if and only if the partial multiplicities of all purely imaginary eigenvalues (if any) of the Hamiltonian matrix $H_{X}$ are two.

b) Suppose that the matrix pair $\left\langle-A^{2}-R S, A R-R A^{T}\right\rangle$ is reachable and that $\imath\left(A R-R A^{T}\right)$ is Hermitian positive semi-definite. Then there exists a real skew-symmetric matrix solution $Y$ of (17) such that the 
closed-loop matrix $A^{2}+R S-\left(A R-R A^{T}\right) Y$ is stable, i.e., all eigenvalues are in the open left half plane if and only if the partial multiplicities of all purely imaginary eigenvalues (if any) of the matrix $H_{Y}$ are two.

Proof. See [15].

Note that if $A, R, S$ are real then the stabilizing solutions of (16) and (17) are also real. The first is shown in [15], while the second follows in the same way using the similarity transformation in (18).

In Theorem 1 and Corollary 2, the existence of the solutions of the Riccati equations in $X$ are contingent upon the negative definiteness of $A R+R A^{T}$. If $A R+R A^{T}$ is indefinite, the existence of the solution to the SDARE is not guaranteed, due to the existence of a conjugate point in the associated differential (game) Riccati equation [3]. Nevertheless, if the solution $X$ to the SDARE exists with $A R+R A^{T}$ indefinite, then it will also be indefinite [16]. A related result holds analogously for $Y$. Continuing, the relationship between $X$ and $Y$ is explicitly derived in the following lemma.

Lemma 3 Suppose there exist a stabilizing solution $X$ to the SDARE and $Y$ to the SSDARE. Then the following equations hold:

$$
\begin{aligned}
& S+A^{T} Y+X(A-R Y)=0 \\
& S+A^{T} X-Y(A-R X)=0 .
\end{aligned}
$$

Proof. Since the matrix $\left[\begin{array}{ll}I & X\end{array}\right]^{T}$ spans the stable invariant subspace of $H_{X}$ and

$$
H_{X} H_{2}=H_{2} H_{Y}
$$

then the matrix

$$
\left[\begin{array}{l}
Y_{1} \\
Y_{2}
\end{array}\right]=\left[\begin{array}{cc}
-A & R \\
S & A^{T}
\end{array}\right]\left[\begin{array}{l}
I \\
X
\end{array}\right]
$$

spans the stable invariant subspace of $H_{Y}$. Thus, the stabilizing solution $Y$ to the SSDARE may be written

$$
\begin{aligned}
Y & =Y_{2} Y_{1}^{-1} \\
& =-\left(S+A^{T} X\right)(A-R X)^{-1} .
\end{aligned}
$$

The analogous result for $H_{Y}$ completes the proof. 


\section{Lemma 4}

a) Suppose $X$ is a real symmetric solution to (16) which stabilizes $A^{2}-$ $R S$. Then a similarity transformation with

$$
T_{X}=\left[\begin{array}{ll}
I & 0 \\
X & I
\end{array}\right]
$$

block triangularizes the matrix $H_{X}$ in (12) with the eigenvalues of the leading $n \times n$ block of the matrix $T_{X}^{-1} H_{X} T_{X}$ stable.

b) Suppose $Y$ is a real skew-symmetric solution of (17) which stabilizes $A^{2}+R S$. Then a similarity transformation with

$$
T_{Y}=\left[\begin{array}{cc}
I & 0 \\
Y & I
\end{array}\right]
$$

block triangularizes the matrix $H_{Y}$ in (13) with the eigenvalues of the leading $n \times n$ block of the matrix $T_{Y}^{-1} H_{Y} T_{Y}$ stable.

Proof. The proof follows by direct substitution, and is given here for later reference.

$$
\begin{aligned}
& T_{X}^{-1} H_{X} T_{X}=\left[\begin{array}{cc}
I & 0 \\
-X & I
\end{array}\right]\left[\begin{array}{cc}
-\left(A^{2}-R S\right) & A R+R A^{T} \\
S A+A^{T} S & \left(A^{2}-R S\right)^{T}
\end{array}\right]\left[\begin{array}{cc}
I & 0 \\
X & I
\end{array}\right] \\
& =\quad\left[\begin{array}{cc}
I & 0 \\
-X & I
\end{array}\right]\left[\begin{array}{cc}
-A^{2}+R S+\left(A R+R A^{T}\right) X & A R+R A^{T} \\
S A+A^{T} S+\left(A^{2 T}-S R\right) X & A^{2 T}-S R
\end{array}\right] \\
& =\left[\begin{array}{cc}
-A^{2}+R S+\left(A R+R A^{T}\right) X & A R+R A^{T} \\
0 & \left(A^{2}-R S-\left(A R+R A^{T}\right) X\right)^{T}
\end{array}\right] .
\end{aligned}
$$

The proof of $b$ ) is analogous.

Lemma 5 Suppose $X$ is a real symmetric solution of (16) which stabilizes $-A^{2}+R S$ and $Y$ is a real skew-symmetric solution of (17) which stabilizes $-A^{2}-R S$. Then the following expressions hold:

$$
\begin{aligned}
-A^{2}-R S+\left(A R-R A^{T}\right) Y & =-(A-R X)(A-R Y) \\
-A^{2}+R S+\left(A R+R A^{T}\right) X & =-(A-R Y)(A-R X)
\end{aligned}
$$


Proof. $H_{X}=H_{2} H_{1}$ and $H_{Y}=H_{1} H_{2}$ where $H_{1}$ and $H_{2}$ are defined as in (11).

The product $T_{X}^{-1} H_{X} T_{X}$ is formed, where $H_{X}$ is expressed as the product $H_{2} T_{Y} T_{Y}^{-1} H_{1}$, and we obtain

$$
\begin{aligned}
& T_{X}^{-1} H_{X} T_{X}=\left(T_{X}^{-1} H_{2} T_{Y}\right)\left(T_{Y}^{-1} H_{1} T_{X}\right) \\
&= {\left[\begin{array}{cc}
I & 0 \\
-X & I
\end{array}\right]\left[\begin{array}{cc}
-A & R \\
S & A^{T}
\end{array}\right]\left[\begin{array}{cc}
I & 0 \\
Y & I
\end{array}\right] \times } \\
& {\left[\begin{array}{cc}
I & 0 \\
-Y & I
\end{array}\right]\left[\begin{array}{cc}
A & -R \\
S & A^{T}
\end{array}\right]\left[\begin{array}{cc}
I & 0 \\
X & I
\end{array}\right] } \\
&=\left[\begin{array}{rr}
-A+R Y & R \\
S+A^{T} Y+X(A-R Y) & X R+A^{T}
\end{array}\right] \times \\
& {\left[\begin{array}{rr}
A-R X & -R \\
S+A^{T} X-Y(A-R X) & Y R+A^{T}
\end{array}\right] . }
\end{aligned}
$$

By Lemma 3,

$$
\begin{aligned}
& S+A^{T} Y+X(A-R Y)=0 \\
& S+A^{T} X-Y(A-R X)=0 .
\end{aligned}
$$

Therefore,

$$
\begin{aligned}
& T_{X}^{-1} H_{X} T_{X}= \\
& =\left[\begin{array}{cc}
-A+R Y & -R \\
0 & X R-A^{T}
\end{array}\right]\left[\begin{array}{cc}
A-R X & -R \\
0 & A^{T}-Y R
\end{array}\right] \\
& =\left[\begin{array}{cc}
-(A-R Y)(A-R X) & -(A-R Y) R-R(A-R Y)^{T} \\
0 & (A-R X)^{T}(A-R Y)^{T}
\end{array}\right] .
\end{aligned}
$$

Analogously it follows that

$$
T_{Y}^{-1} H_{Y} T_{Y}=\left[\begin{array}{cc}
-(A-R X)(A-R Y) & -(A-R X) R+R(A-R X)^{T} \\
0 & (A-R Y)^{T}(A-R X)^{T}
\end{array}\right] .
$$

The previous lemma illustrates that the product $-(A-R X)(A-R Y)$ is stable. We need, however, that the products $-(A-R X)(A-R X)$ and $-(A-R Y)(A-R Y)$ are stable. This is equivalent to the statement that all 
the eigenvalues of $(A-R X)$ and $(A-R Y)$, respectively, lie in the interior of the union of the damped and anti-damped regions of the complex plane.

Theorem 6 Suppose that $X$ is a real symmetric dampening solution of (16) and $Y$ is the real skew-symmetric dampening solution of (17). Then the closed-loop matrices $A-R X$ and $A-R Y$ have eigenvalues within the closure of the union of the damped and anti-damped regions of the complex plane (excluding the point 0 ).

Proof. The proof employs a standard result from Lyapunov stability theory [17], namely that:

$\hat{A}$ is stable if and only if there exists

$$
M=M^{T}>0 \text { such that } \hat{A}^{T} M+M \hat{A}<0 .
$$

Define $S_{\epsilon}=S+\epsilon I$. Then, since we have assumed that $S \geq 0$, we have that $S_{\epsilon}>0$. We first prove the result for the case that we use the weighting matrix $S_{\epsilon}$.

Let a candidate for $M$ be

$$
M=X R X-A^{T} X-X A-S_{\epsilon} .
$$

If $-(A-R X)^{2}$ is stable then the spectrum of $A-R X$ is in the required region, so it suffices to show that

$$
\begin{array}{cc}
P=-(A-R X)^{2 T} M-M(A-R X)^{2} & <0 \\
M & >0 .
\end{array}
$$

By Lemma 3 we have

$$
A^{T} X+S_{\epsilon}=Y(A-R X),
$$

and thus

$$
\begin{aligned}
M & =-A^{T} X-X(A-R X)-S_{\epsilon} \\
& =-(X+Y)(A-R X) .
\end{aligned}
$$

Further, by noting that $X=X^{T}, Y=-Y^{T}$, and $M=M^{T}$, and therefore

$$
M=-(A-R X)^{T}(X-Y),
$$

it follows that

$$
P=(A-R X)^{T}\left((A-R X)^{T}(X+Y)+(X-Y)(A-R X)\right)(A-R X)
$$


By using (31), it is possible to further simplify $P$ :

$$
\begin{aligned}
P & =(A-R X)^{T}\left((A-R X)^{T}(X+Y)+(X-Y)(A-R X)\right)(A-R X) \\
& =(A-R X)^{T}\left((A-R X)^{T} X+X(A-R X) \ldots\right. \\
& \left.-X A-A^{T} X-2 S_{\epsilon}\right)(A-R X) \\
& =-2(A-R X)^{T}\left(X R X+S_{\epsilon}\right)(A-R X)<0 .
\end{aligned}
$$

Here we have made use of the fact that $(A-R X)(A-R Y)$ stable implies in particular that $A-R X$ is non-singular.

Proceeding to the second proposition in (30), we note that

$$
\begin{aligned}
Q & =-(A-R X)^{T}\left((A-R Y)^{T} M-M(A-R Y)\right)(A-R X) \\
& =(A-R X)^{T}\left((A-R Y)^{T}(X+Y)+(X-Y)(A-R Y)\right)(A-R X) \\
& =-2(A-R X)^{T}\left(Y R Y^{T}+S_{\epsilon}\right)(A-R X)<0 .
\end{aligned}
$$

Since $Q$ is negative definite and $-(A-R Y)(A-R X)$ is stable, $M$ must be positive definite [6]. Thus, it follows that $-(A-R X)^{2}$ is stable and hence all eigenvalues of $A-R X$ are in the union of the damped and the anti-damped regions of the complex plane for all $\epsilon \geq 0$. Letting $\epsilon$ tend to zero, we obtain by continuity that all eigenvalues of $A-R X$ are in the closure of the damped and the anti-damped regions of the complex plane, and from the fact that $A-R X$ is non-singular also in the limit, we have guaranteed that $A-R X$ is as required.

By applying the same techniques for the closed-loop matrix $A-R Y$, and letting $M=Y R Y+A^{T} Y-Y A-S$, the proof is complete.

Note that we obtain that all the eigenvalues are in the closure of the damped and anti-damped regions excluding the point zero. If we want to guarantee that all eigenvalues are in the interior of these regions, we can achieve this either by choosing $S>0$ or by providing an appropriate stabilizability and detectability assumption. This is well known for standard stabilization problems and carries over in a canonical way.

Remark 1 In practice, the solutions $X$ and $Y$ of (16) and (17) are computed by simultaneously computing a basis for the stable invariant subspaces of $H_{2} H_{1}$ and $H_{1} H_{2}$ via the periodic Schur algorithm [5, 9, 10], which is a numerically robust method. Note that in general the computation of the periodic Schur decomposition will yield eigenvalues of the closed-loop system in the union of damped and anti-damped regions. To move the poles from 
the anti-damped region into the damped region one may solve a degenerate Riccati equation, (see e.g. [8]). This is well-known to reflect the eigenvalues at the imaginary axis. If we compute the symmetric, stabilizing solution of

$$
(A-R X)^{T} U+U(A-R X)-U R U=0
$$

or the skew-symmetric stabilizing solution of

$$
(A-R Y)^{T} V+V(A-R Y)-Y R Y=0,
$$

then those eigenvalues of $A-R X$ and $A-R Y$ in the anti-damped region of the complex plane will be reflected back across the imaginary axis into the damped region, becoming the eigenvalues of $A-R(X+U)$ and $A-R(Y+V)$ respectively.

Remark 2 Taking the sum of the symmetric solutions $X$ and $U$ of (16) and (32), one obtains the residual equation

$$
S_{X U}:=(X+U) R(X+U)-A^{T}(X+U)-(X+U) A .
$$

Once $S_{X U}$ has been formed, one can use (14) to compute $W_{X U}=(X+U)$ directly. This provides a means of checking the sensitivity of the feedback via existing theorems concerning eigenvalue sensitivity of standard Riccati equations [12]. It also provides valuable information concerning the required state weighting to achieve a dampening controller.

Although the symmetric dampening solution seems to be more attractive theoretically, numerical experiments seem to indicate that the nonsymmetric feedback $Y+V$ from (17) and (33) sometimes, but not always, produces less sensitive feedbacks. One heuristic for determining the least sensitive feedback is to compute the norms of the two feedbacks $\|R(X+U)\|$ and $\|R(Y+V)\|$. The feedback of smaller norm tends to be less sensitive.

\section{Algorithmic Details}

We now describe the details of the numerical algorithm to compute the symmetric dampening controllers.

\section{Algorithm 1 \\ $\operatorname{INPUT}(A, B, C, \sigma, \rho)$}


\% A: system matrix

\% B: input matrix

$\% \quad C:$ output matrix

$\% \quad \sigma:$ shift from imaginary axis

$\% \quad \rho:$ small shift in preprocessing step

$\%$

Compute $\nu=\max \{|\lambda|, \lambda \in \Lambda(A)$ i.e., the spectrum of $A\}$.

Scale $A \leftarrow A / \nu, B \leftarrow B / \sqrt{\nu}, C \leftarrow C / \sqrt{\nu}$.

Form $R=B B^{T}$.

$\%$

$\% \quad$ Preprocessing Step

$\% \quad$ This step is a heuristic which lowers the norm of $X$

$\% \quad$ (See Example 1 in the Numerical Examples section for details)

$\%$

Compute the stabilizing solution of (15), where $S=C^{T} C$ and $\sqrt{\epsilon}<\rho \ll 1$, with $\epsilon$ the machine epsilon.

$\%$

$\% \quad$ End Preprocessing Step

$\%$

Form $H_{1}$ and $H_{2}$ as in (11) with $A=A_{Z}=A-R Z$ and $S=0$.

Compute real orthogonal matrices $Q_{X}$, and $Q_{Y}$, using the periodic Schur decomposition, with inputs $H_{1}$ and $H_{2}$, such that $Q_{X}^{T} H_{2} H_{1} Q_{X}=\Delta_{X}$ and $Q_{Y}^{T} H_{1} H_{2} Q_{Y}=\Delta_{Y}$ are block upper triangular with stable blocks in the upper left corner.

Partition $Q_{X}=\left[\begin{array}{ll}Q_{11} & Q_{12} \\ Q_{21} & Q_{22}\end{array}\right]$ and $Q_{Y}=\left[\begin{array}{ll}\tilde{Q}_{11} & \tilde{Q}_{12} \\ \tilde{Q}_{21} & \tilde{Q}_{22}\end{array}\right]$

Compute $X=Q_{21} Q_{11}^{-1}$ and $Y=\tilde{Q}_{21} \tilde{Q}_{11}^{-1}$. 
Form $A_{Z X}=A-R(Z+X)$ and $A_{Z Y}=A-R(Z+Y)$.

Compute the stabilizing solutions $U$ and $V$ to the Riccati equations:

$U\left(A_{Z X}-\sigma I\right)+\left(A_{Z X}^{T}-\sigma I\right) U-U R U=0$ and

$V\left(A_{Z Y}-\sigma I\right)+\left(A_{Z Y}^{T}-\sigma I\right) V-V R V=0$.

Form $A_{Z X U}=A-R(Z+X+U)$ and $A_{Z Y V}=A-R(Z+Y+V)$.

Scale $A_{Z X U} \leftarrow \nu A_{Z X U}, A_{Z Y V} \leftarrow \nu A_{Z Y V}$

$\operatorname{OUTPUT}\left(A_{Z X U}, A_{Z Y V}, U, V, X, Y, Z\right)$.

\section{Numerical Examples}

In this section we give some numerical examples to illustrate the results discussed in the previous sections.

Example 1 In this example, we demonstrate Algorithm 1 performed under complex arithmetic for scalar inputs. Let

$$
\begin{aligned}
& A=a+\imath \alpha \\
& B=b+\imath \beta .
\end{aligned}
$$

In complex arithmetic, we replace the transposition operation in (16) and (17) with the complex-conjugation operation, and we seek to find the complex scalars $X$ and $Y$ which form the optimal closed-loop scalars

$$
\begin{aligned}
\tilde{A}_{X} & =A-B B^{H} X \\
& =a+\imath \alpha-\left(b^{2}+\beta^{2}\right) X
\end{aligned}
$$

and

$$
\begin{aligned}
\tilde{A}_{Y} & =A-B B^{H} Y \\
& =a+\imath \alpha-\left(b^{2}+\beta^{2}\right) Y .
\end{aligned}
$$

It may be easily confirmed, with $S=0$ that

$$
\begin{gathered}
X=\frac{\left(a^{2}-\alpha^{2}\right)}{a\left(b^{2}+\beta^{2}\right)}, \quad Y=\frac{-\imath\left(a^{2}-\alpha^{2}\right)}{\alpha\left(b^{2}+\beta^{2}\right)}, \\
\tilde{A}_{X}=\frac{\alpha}{a}(\alpha+\imath a), \tilde{A}_{Y}=\frac{a}{\alpha}(\alpha+\imath a) .
\end{gathered}
$$


As expected $\Lambda\left(\tilde{A}_{X} \tilde{A}_{Y}\right)=\Lambda\left(-A^{2 H}\right)$. It is interesting to note that with an exception of a real scaling the closed-loop eigenvalues of $\tilde{A}_{X}$ and $\tilde{A}_{Y}$ are merely reflected across the $(1 \pm \imath) \omega$ axis, i.e., the diagonal axes in the complex plane. Further, note that the real scaling $\frac{\alpha}{a}$ is unbounded as $A$ approaches the imaginary axis, which in turn produces an unbounded damped Riccati solution $X$. Performing this same experiment in real arithmetic on matrices

$$
\begin{aligned}
& A=\left[\begin{array}{cc}
a & \alpha \\
-\alpha & a
\end{array}\right] \\
& B=\left[\begin{array}{cc}
b & \beta \\
-\beta & b
\end{array}\right]
\end{aligned}
$$

produces the exact analogous result, which motivates the use of the SHARE in the "preprocessing" step of Algorithm 1.

Example 2 In this example, it is demonstrated how Algorithm 1 may be used to restrict the poles of a closed-loop system to a damped and well stabilized convex region within the left-hand plane. A total of 256 reachable and observable linear time-invariant systems $[A, B, C]$ are randomly generated with state dimension $n=12$, input dimension $m=2$ and output dimension $p=2$. The matrices along with $\rho=0.05$ and $\sigma=0.5$ are the inputs to Algorithm 1. The eigenvalues of the closed-loop matrices $A_{Z X U}$ and $A_{Z Y V}$ from the output of Algorithm 1 are plotted in Figure 2.

Example 3 In this example, we dampened a system of springs, dashpots, and masses with two inputs, as shown by Figure 3 . The system is modeled by the following time-invariant linear system

$$
\begin{gathered}
A=\left[\begin{array}{cc}
0 & I \\
M^{-1} K & -M^{-1} D
\end{array}\right], B=\left[\begin{array}{l}
0 \\
\tilde{B}
\end{array}\right], \\
C=c \\
{\left[\begin{array}{ll}
I & 0 \\
0 & I
\end{array}\right]}
\end{gathered}
$$


Eigenvalues with the Symmetric Damped Riccati Solution

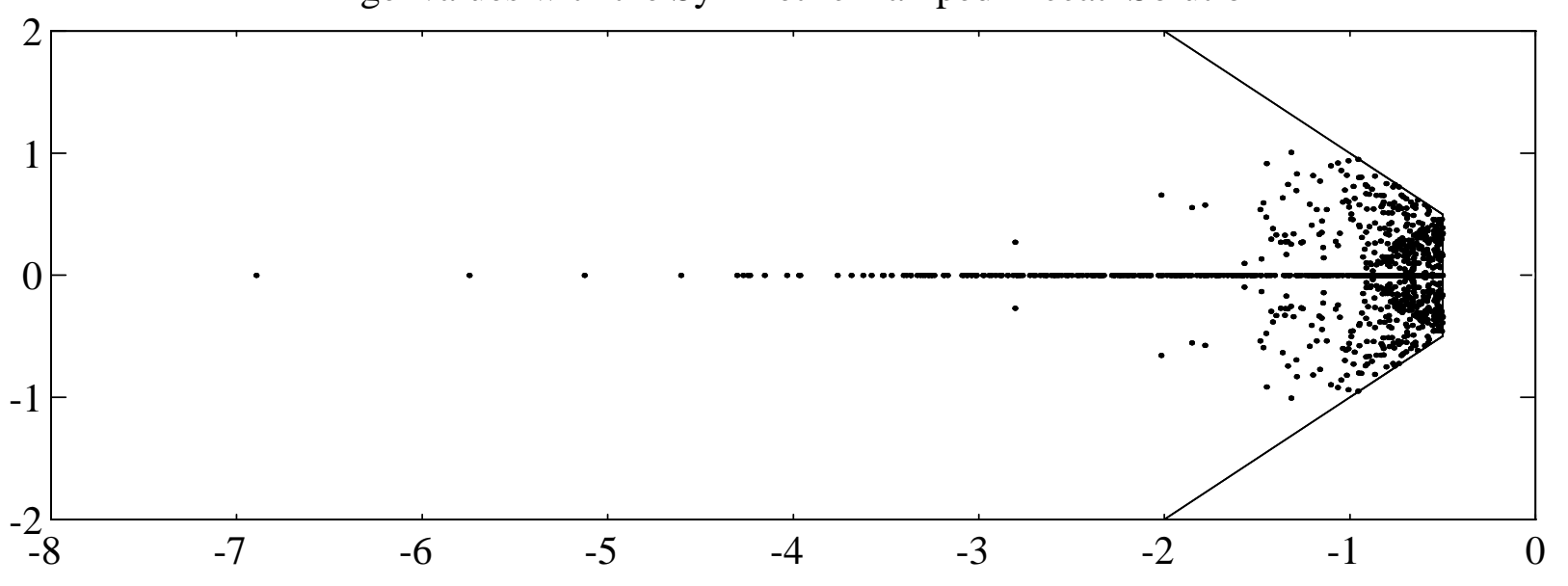

Eigenvalues with the Non-Symmetric Damped Riccati Solution

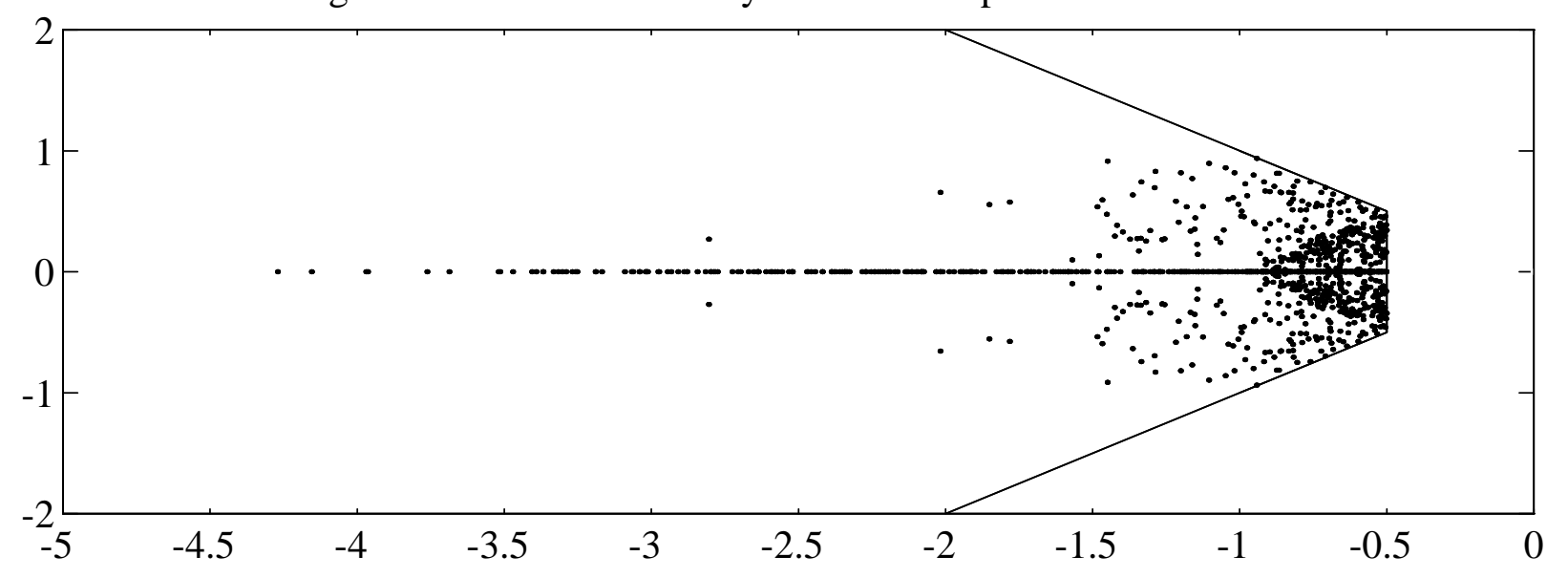

Figure 2: Closed-Loop Eigenvalues for Randomly Generated Systems 

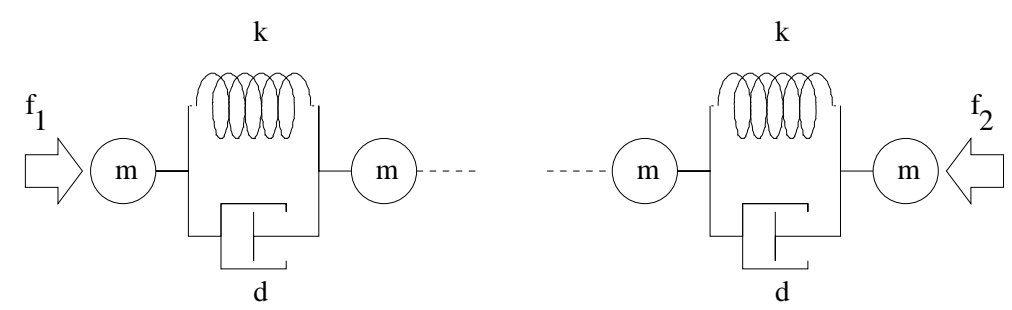

Figure 3: Coupled Spring Experiment

where $M=\mu I, D=\delta I$,

$$
K=\kappa\left[\begin{array}{ccccccc}
-1 & 1 & 0 & \cdots & 0 & 0 & 0 \\
1 & -2 & 1 & \cdots & 0 & 0 & 0 \\
0 & 1 & -2 & \cdots & 0 & 0 & 0 \\
\vdots & \vdots & \ddots & \ddots & \ddots & \vdots & \vdots \\
0 & 0 & 0 & \cdots & -2 & 1 & 0 \\
0 & 0 & 0 & \cdots & 1 & -2 & 1 \\
0 & 0 & 0 & \cdots & 0 & 1 & -1
\end{array}\right] \quad, \quad \tilde{B}=\left[\begin{array}{cc}
1 & 0 \\
0 & 0 \\
0 & 0 \\
\vdots & \vdots \\
0 & 0 \\
0 & 0 \\
0 & -1
\end{array}\right] .
$$

In this experiment, we demonstrate the efficiency of Algorithm 1 in producing dampening controllers for systems of large dimension. In this case, $n=60$ (thirty springs), $\mu=4, \kappa=1$, and $\delta=4$. The eigenvalues of the closed-loop system are shown in Figure 4.

Example 4 In this example, we show how the eigenvalues of the closedloop system may be restricted to a tighter region of the left half-plane. This is accomplished in part by replacing the Hamiltonian system $H_{X, 2}=H_{X}=$ $\mathrm{H}_{2} \mathrm{H}_{1}$ in (12) with systems of higher periodicity. Two such Hamiltonian systems are examined:

$$
H_{X, 3}=H_{2} H_{1} H_{1}
$$

and

$$
H_{X, 4}=H_{2} H_{1} H_{1} H_{1},
$$

with $H_{1}$ and $H_{2}$ given by (11), respectively. Although we focus in this example on periodic Hamiltonian systems of period three and four, our intent is to motivate the general observation that Hamiltonian systems of period $p$ may be used to produce feedbacks by which the eigenvalues of the attendant closed-loop system are contained within regions bounded by the stability cones that subtend the angle $180 / p$ degrees, as shown by Figure 5 . 

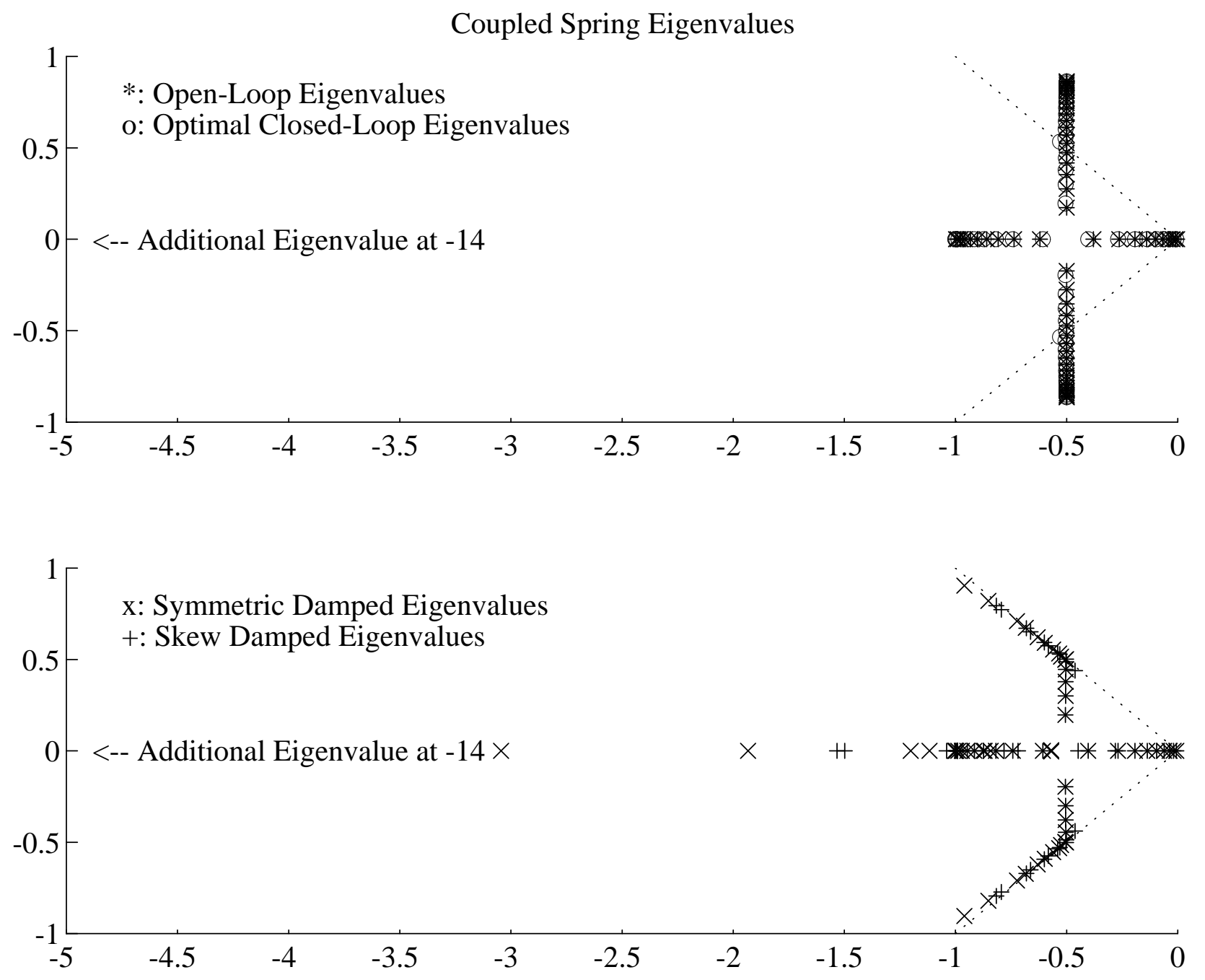

Figure 4: Damped Eigenvalues 


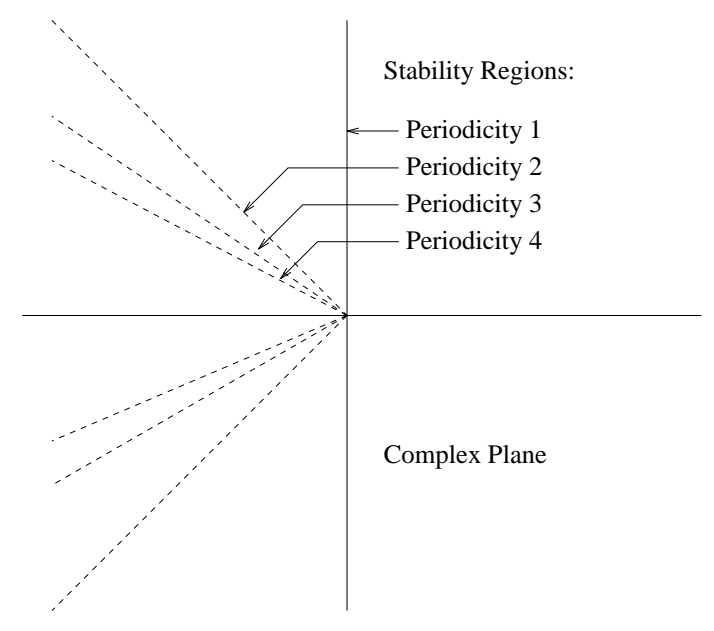

Figure 5: Stability Regions versus Hamiltonian Periodicity

As with the case for period two, the Hamiltonian matrices $H_{X, p}$ will give rise to symmetric matrices $X_{p}$, which are symmetric solutions to Riccati equations similar to (16). For the sake of brevity, only the feedbacks which use the symmetric solutions $X_{p}$ are examined in this example, but there exist other solutions associated with different permutations of the order of $H_{1}$ and $H_{2}$ in (37) and (38). For example, the three Hamiltonian systems of period three produce three Riccati solutions: one symmetric and two non-symmetric, while the four Hamiltonian systems of period four produce four Riccati solutions: one symmetric, one skew symmetric and two nonsymmetric.

The procedure for computing dampening feedbacks follows along the same line as that in Algorithm 1, namely that first a stabilizing feedback $Z$ is produced with $S=C^{T} C$ and $\rho$ small. The matrix $X_{3}$ is computed from the periodic Schur decomposition of the implicit periodic map $H_{X, 3}$ with $A=A_{Z}$ and $S=0$, in complete analogy to Algorithm 1. In the case with Hamiltonian periodicity $p=4$, an extra step is required. With $p=4$, an eigenvalue $\lambda=\rho^{i \theta}$ of the closed-loop matrix $A_{Z X, 4}=A-B B^{T}\left(Z+X_{4}\right)$ may be contained in the regions where $-\frac{\pi}{8} \leq \theta \leq \frac{\pi}{8}, \frac{3 \pi}{8} \leq \theta \leq \frac{5 \pi}{8}, \frac{7 \pi}{8} \leq \theta \leq \frac{9 \pi}{8}$, and $-\frac{5 \pi}{8} \leq \theta \leq-\frac{3 \pi}{8}$. If the periodic step of Algorithm 1 is repeated with Hamiltonian periodicity $p=2$, however, the resulting feedback (using Riccati solution $X_{2}$ ) reflects the eigenvalues in the regions near the imaginary axis across the $(1+\imath) \omega$ and the $(1-\imath) \omega$ axes, thus placing them the region indicated by Figure 5 . 
Eigenvalues via Symmetric Damped Riccati Solution, Period 3

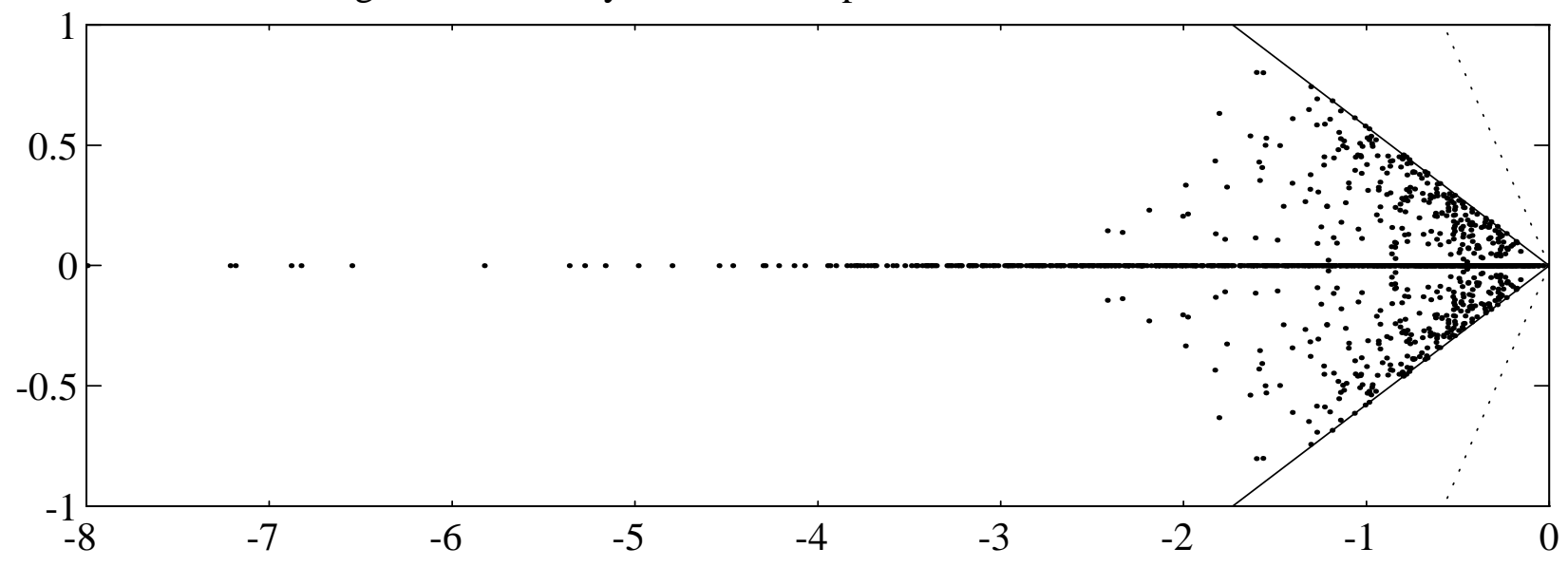

Eigenvalues via Symmetric Damped Riccati Solution, Period 4

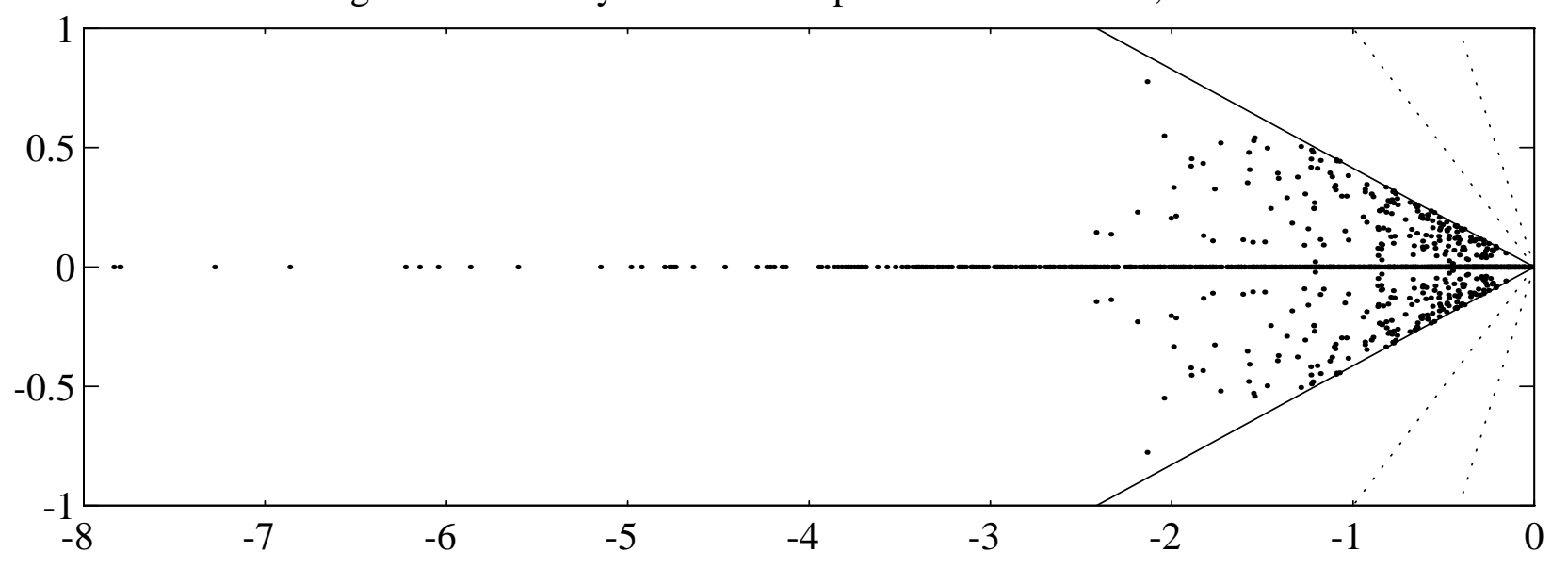

Figure 6: Closed-Loop Eigenvalues for Randomly Generated Systems 
As in Example 1, a total of 256 reachable and observable linear timeinvariant systems $[A, B, C]$ are randomly generated, however, with state dimension $n=7$, input dimension $m=4$ and output dimension $p=3$, and shift $\sigma=0$. The eigenvalues of the closed-loop matrices $A_{Z X_{2} U}=$ $A-B B^{T}\left(Z+X_{3}+U\right)$ and $A_{Z X_{4} X_{2} U}=A-B B^{T}\left(Z+X_{4}+X_{2}+U\right)$ plotted in Figure 6.

\section{Conclusion}

In this paper we have proposed a new method to produce a dampening controller. It promises to be an efficient and numerically reliable method to restrict the eigenvalues of a closed-loop state matrix to relatively elaborate regions in the left half-plane, without resorting to pole-placement.

Still, many issues remain open, and are presently being investigated. Among these are standard analyses of the sensitivity of the eigenvalues of the closed-loop state matrix, scalings of the Hamiltonian to produce optimal results. Also, we are aware of different parameterized formulations of the periodic Hamiltonian system in complex arithmetic which also produce excellent dampening controllers. We have also observed experimentally that convex-combinations of the feedbacks discussed in this paper remain stable. These results have not been reported in this paper for the sake of brevity; they will be given in another manuscript. Nevertheless, the results contained herein provide the control engineer with a great deal more flexibility in designing a controller for time-invariant systems described in state-space form.

\section{References}

[1] Anderson, B.D.O. and J.B. Moore, Linear Optimal Control, PrenticeHall, Englewood Cliffs, NJ, 1971.

[2] Başar, T., and Olsder, G.J., Dynamic Noncooperative Game Theory Academic Press, New York, 1982.

[3] Başar, T., "Generalized Riccati Equation in Dynamic Games", The Riccati Equation, S. Bittanti, A.J. Laub and J.C. Willems eds., Springer Verlag, (1991), pp. 11-52. 
[4] Bittanti, S., Laub, A.J., and Willems, J.C., The Riccati equation Springer-Verlag, Berlin, 1991.

[5] Bojanczyk, A., G.H. Golub, and P. Van Dooren, "The Periodic Schur Decomposition. Algorithms and Applications," Proc. SPIE Conf., volume 1770, pp. 31-42, 1992.

[6] Gantmacher, F.R., The Theory of Matrices, volume II, Chelsea, New York, 1959.

[7] He, C., Laub, A.J., Mehrmann, V., Placing Plenty of Poles is Pretty Preposterous, Preprint: Fakultät für Mathematik, TU Chemnitz-Zwickau, 1995.

[8] He, C., Mehrmann, V., Stabilization of Large Lincar Systems, Proceedings of the IEEE Workshop on Computer Intensive Methods in Control and Signal Processing, Prague, September 1994.

[9] Hench, J.J., Numerical Methods for Periodic Linear Systems, PhD thesis, University of California, Santa Barbara, Electrical and Computer Engineering Dept., September 1992.

[10] Hench, J.J. and A.J. Laub, "Numerical Solution of the Discrete-Time Periodic Riccati Equation," IEEE Trans. Auto. Control, 39 (1994), pp. $1197-1210$.

[11] Kalman, R.E., "Contributions to the Theory of Optimal Control," Boletin Sociedad Matematica Mexicana, 5(1960), pp. 102-119.

[12] Kenney, C. and G. Hewer, "The Sensitivity of the Algebraic and Differential Riccati Equations," SIAM J. Control Opt., 28(1990), pp. 50-69.

[13] Kučera, V., "A Contribution to Matrix Quadratic Equations," IEEE Trans. Auto. Control, AC-17 (1972), pp. 344-347.

[14] Laub, A.J., "A Schur Method for Solving Algebraic Riccati Equations," IEEE Trans. Auto. Control, AC-24 (1979), pp. 913-921, (see also Proc. 1978 CDC (Jan. 1979), pp. 60-65).

[15] Lancaster, P. and Rodman, L., "Existence and Uniqueness Theorems for the Algebraic Riccati Equation", Int. J. Control, 32 (1980), pp. 285309 . 
[16] Lancaster, P. and Rodman, L., "Solutions of the Continuous and Discrete Time Algebraic Riccati Equation: A Review", The Riccati Equation, S. Bittanti, A.J. Laub and J.C. Willems eds., Springer Verlag, (1991), pp. 11-52.

[17] Lancaster, P. and Tismenetsky, M., "The Theory of Matrices", 2nd ed., Academic Press, Orlando, Florida (1985).

[18] Mehrmann, V. The Autonomous Linear Quadratic Control Problem, Theory and Numerical Solution, Springer-Verlag, Heidelberg, 1991.

[19] Pappas, T., A.J. Laub, and N.R. Sandell, "On the Numerical Solution of the Discrete-Time Algebraic Riccati Equation," IEEE Trans. Auto. Control, AC-25 (1980), pp. 631-641.

[20] Potter, J.E., "Matrix Quadratic Solutions," SIAM J. Appl. Math., 14 (1966), pp. 496-501.

[21] Roberts, J.D., "Linear Model Reduction and Solution of the Algebraic Riccati Equation by use of the Sign Function," Int. J. Control, 32 (1980), pp. 677-687. (Reprint of Technical Report No. TR-13, CUED/B - Control, Cambridge University, Dept. of Engineering, 1971.) 